\section{UNIVERSAL ISOTROPY}

\section{Essential Requirement for Life?}

by our Cosmology Correspondent

Two fundamental problems each provide common cause for discussion among cosmologists. Why is the Universe isotropic? Is the Universe bound or unbound? Now, Collins and Hawking (University of Cambridge) suggest that the isotropy of the Universe is a direct result of its expansion with a velocity just on the border between infinite expansion with more than the escape velocity and eventual collapse under the gravitational influcnce of the whole system. Further, they argue that galaxies can only grow in such a universe, and that therefore the presence of life is a direct consequence of the isotropy of the Universe-or at least, the two have a common cause (Astrophys. J., 180, 317 ; 1973).

To take the second common question first, the problem of the Universe being bound or unbound is simply one of the velocity of expansion of the Universe in relation to the escape velocity (throughout this discussion a big-bang origin is assumed). The amount of matter in the Universe inferred from observations of bright galaxies is not sufficient to close the system and prevent ultimate expansion to infinity. But there could well be enough dark matter-cool gas, black holes, or even neutrinos-to do the job. Because this dark matter is by definition invisible from Earth, there is plenty of scope for discussion.

Too little is known about the Universe for the discussion to be closed yet. But another topic which has now been closed for discussion is the question of the isotropy of the Universe. With the discovery of the microwave background radiation, and the growing conviction that this is indeed a relic of the big-bang, ever more sophisticated measurements have shown that the radiation, and hence the Universe, is isotropic. One of the most recent measurements, reported by Parijskij, sets as a limit that there are no fluctuations of the blackbody background above the $0.8 \times 10^{-4} \mathrm{~K}$ r.m.s. level at $2.8 \mathrm{~cm}$ on scales of 3 arc min to 1 arc deg (Astrophys. J. Lett., 180, L47; 1973). The temperature of the radiation is about $2.7 \mathrm{~K}$, so the accuracy of these measurements is indeed good.

Given that the Universe is isotropic, then, what can be deduced about its origin and other parameters? One diffculty which arises is that if the Universe is homogeneous and isotropic-like the Robertson-Walker model-how do local inhomogeneities such as galaxies and stars grow up? As Collins and Hawking point out, the usual way in which this problem has been tackled is by studying the growth of small perturbations in a Robertson-Walker back- ground. But perturbations grow disappointingly slowly in a Robertson-Walker universe, and this has led, for example, to suggestions that the original state of our Universe was one of extreme chaos and irregularity. Certainly not all initial conditions would lead to a universe like the one in which we live.

Even worse, initid inhomogeneities should lead to eventual anisotropy. Collins and Hawking have tackled the problem from a different point of view, adopting the philosophy attributed to Dicke and Carter that there is not just one universe but an infinite ensemble of universes, so that all the peculiarities in which mathematicians delight can have a physical being. Only those universes which contain galaxies can have intelligent life, say Collins and Hawking, and galaxies will not occur in highly anisotropic universes.

The philosophy is supported by the mathematics. It turns out that spatially homogeneous model universes can be divided into three classes: those which expand at below the escape velocity and eventually collapse ; those which expand at just about the escape velocity; and those which expand with more than the escape velocity. Those in the first class do not exist long enough to become isotropic, and in any case there is probably insufficient time for galaxies to grow in them during the expansion phase. The third class do not tend towards isotropy, according to Collins and Hawking, and in any case initial perturbations will not grow in density because they too will be expanding more rapidly than the local escape velocity.

That leaves a small class of models, not unlike Robertson-Walker models, expanding at the critical escape velocity and tending towards isotropy. Only in these universes can initial perturbations grow into galaxies. As Collins and Hawking put it, the answer to the question "Why is the Universe isotropic?" is "Because we are here". That seems to be putting the cart before the horse; I would prefer to answer the question "Why are we here?" by saying "Because the Universe is isotropic". But however one splits that particular hair, the point is that we are here, the Universe is isotropic, and that such a situation can indeed be explained with sensible cosmological assumptions.

\title{
Is Cyg X-3 Similar to Sco X-1?
}

THE Cyg X-3 radio flare "closely resembles outbursts which are frequently observed to take place in radio galaxies and quasars", write Braes et al. in next Monday's Nature Physical Science (April 2). The energies involved in this outburst from a small source within our Galaxy are, of course, several orders of magnitude less than those associated with the violent extragalactic events. But Cyg $\mathrm{X}-3$ is only about $10 \mathrm{kpc}$ away from Earth, and its angular size $(0.01$ arc s on September 24) implies an expansion velocity of $0.2 \mathrm{c}$. Several active extragalactic sources have shown evidence of similarly high expansion velo. cities, and indeed such sources often consist of at least two components. Braes et al. point out that it is possible that an event similar to that observed in Cyg X-3 last year could have produced the triple structure seen in the radio source associated with Sco $\mathrm{X}-1$.

All this speculation arises from an analysis of detailed observations of Cyg X-3 during September and October 1972 at 1.4 and $2.7 \mathrm{GHz}$, using the Westerbork synthesis telescope and the Effelsberg $100-\mathrm{m}$ instrument. The observations provide further support for the view that Cyg $X-3$ lies either within or behind the hydrogen emission feature at $-68 \mathrm{~km} \mathrm{~s}^{-1}$ (see also Lauqué et al., Nature Physical Science, 241, 94 ; 1973). Together with kinematic evidence, this means that the distance to the source is at least $10 \pm 1.5 \mathrm{kpc}$; the radio observa- tions suggest that $\mathrm{Cyg} \mathrm{X}-3$ is not much further away than this.

More details of the multiple structure of the radio flare are also presented by Braes et al. The first event, which lasted from September 3 to 10, was a simple outburst consistent with an expanding synchrotron source. The second event was more complex and violent, with three distinct maxima. The first flare (September 3) was similar to the first component of the second event, but the second and third components of that event lasted twice as long and cannot be explained in terms of a repetition of the same process with different energies on the basis of the data available so far. It is not yet possible to say whether the longer durations are a result of smooth injection or repeated discrete injection events. Cyg $X-3$ now seems to have returned to its normal state.

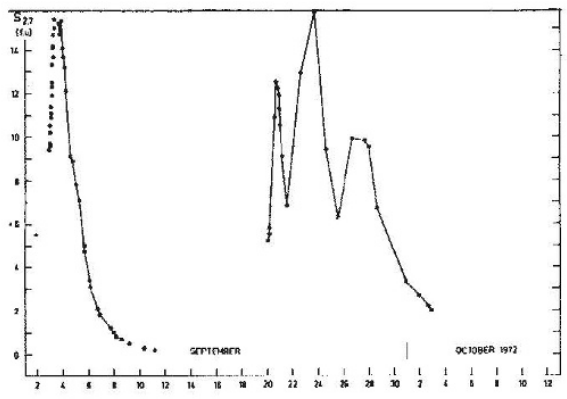

Observations of $\mathrm{Cyg} X-3$ flares at 2.7 GHz with the Max Planck $100-\mathrm{m}$ telescope at Effelsberg. 